Meta

Journal des traducteurs

Translators' Journal

\title{
Étiologie de certains brouillages terminologiques de la langue médicale
}

\section{Amal Jammal}

Volume 34, numéro 4, décembre 1989

URI : https://id.erudit.org/iderudit/003251ar

DOI : https://doi.org/10.7202/003251ar

Aller au sommaire du numéro

Éditeur(s)

Les Presses de l'Université de Montréal

ISSN

0026-0452 (imprimé)

1492-1421 (numérique)

Découvrir la revue

Citer cet article

Jammal, A. (1989). Étiologie de certains brouillages terminologiques de la

langue médicale. Meta, 34(4), 764-769. https://doi.org/10.7202/003251ar d'utilisation que vous pouvez consulter en ligne.

https://apropos.erudit.org/fr/usagers/politique-dutilisation/ 


\section{ÉTIOLOGIE DE CERTAINS BROUILLAGES TERMINOLOGIQUES DE LA LANGUE MÉDICALE}

Nous sommes revenus au temps de Babel; mais on ne travaille plus à un monument commun de confusion : chacun bâtit sa tour à sa propre hauteur.

François René de Chateaubriand

Une démarche diagnostique qui tente de dépister la cause d'un brouillage terminologique donné se heurte souvent à des difficultés complexes. Le trouble auquel ce brouillage est imputable peut être, en effet, de nature notionnelle, d'origine purement terminologique ou encore d'origine notionnelle et terminologique.

Nous choisirons, pour illustrer notre propos, un terme de la langue de l'allergie, emprunté à l'anglais: "prick test», parce qu'il a le douteux privilège de cumuler, à lui seul, plusieurs facteurs de confusion. Le problème a été mis au jour lorsque des auteurs francophones, voulant utiliser l'équivalent de ce terme dans leurs écrits, se sont trouvés devant un vide terminologique, ou encore devant une équivalence proposée par Gladstone ${ }^{1}$, qu'ils auront rejetée parce que, comme nous le verrons plus loin, elle ne recouvre pas la réalité. Les solutions adoptées alors ont varié considérablement selon le gré de chacun, allant de l'approximation ou de l'assimilation jusqu'à l'emprunt pur et simple du terme anglais, assorti, il est vrai, de la mise entre guillemets. Pour les terminologues que ces ersatz de solutions ne satisfaisaient pas et qui ont essayé de démêler cet écheveau de notions et de termes, le problème s'est révélé multifactoriel.

Pour en montrer toute la complexité, nous avons éprouvé le besoin de reproduire ici ce que disent du test lui-même différents auteurs, anglophones et francophones. On pourra ainsi constater l'existence de divergences à la fois quant à la couche de la peau concernée par le test et quant à la technique utilisée pour l'application de ce test.

Ainsi, certains auteurs spécifient que la substance allergène doit être injectée dans l'épiderme; d'autres recommandent de l'injecter dans le derme. On peut lire en effet, dans Allergy: An International Textbook, ce qui suit:

Prick tests... introduce a volume of $10^{-6}$ ml into the epidermis ${ }^{2}$.

W.P. Jackson et R. Cerio, dans Color Atlas of Allergy, confirment :

... skin-prick tests differ from... intracutaneous tests... Intracutaneous tests in which allergens are injected more deeply into the skin, are reproducible and have an occasional role; but they are in general less specific, less well authenticated and more hazardous than prick test... ${ }^{3}$

Plus loin, ces auteurs décrivent ainsi la technique elle-même:

Skin prick testing: The skin is cleaned, prick sites are marked and a small drop of each allergen solution is placed on the skin. A lance is introduced through the drop to a depth of about $1 \mathrm{~mm}$ into the skin, and pulled out raising the skin in the process. The skin should not bleed... ${ }^{4}$

Jusque là, tout est harmonie: le même descripteur se dégage des trois énoncés: le «prick test» (que l'on nous permette pour quelque temps encore de nous servir de l'emprunt) est un test épidermique. En fait, même si dans les deuxième et troisième énoncés ce descripteur n'est pas explicitement indiqué, il l'est implicitement:

1) par le fait qu'il se différencie du test intradermique (intracutaneous test) et 
2) par la recommandation «skin should not bleed». Car, comme on le sait, seul l'épiderme n'est pas vascularisé ; le derme, qui l'est abondamment, saigne quand on le pique.

Autrement dit, sans l'ombre d'un doute, le «prick test» a lieu dans l'épiderme. Ce qui infirme déjà l'équivalence proposée par Gladstone, soit prick test $=$ intradermoréaction 1 .

Cependant, le premier son de cloche discordant concernant la couche de la peau dans laquelle est injectée la substance nous vient du Dictionnaire des constantes biologiques, dans lequel on peut lire :

Une autre technique consiste à pratiquer une fine piqûre du derme (sic) au travers de la goutte d'extrait allergénique («prick test») $)^{5}$.

Ailleurs, la confusion ne concerne pas la couche de la peau, mais la technique d'inoculation de la substance incriminée. En effet, alors que l'appellation même du test implique un piqûre (prick), on peut lire dans les Allergies, la fin d' une énigme:

Les tests cutanés consistent à introduire un allergène dans la peau, soit par voie intradermique (intradermo-réaction), soit à travers l'épiderme (prick-test) au moyen d'une scarification $(\text { re-sic ! })^{6}$.

Or, le test par scarification (qui, par ailleurs, se dit scratch test en anglais) - ou encore cuti-réaction en français - fait appel à une technique totalement différente, dans laquelle on pratique, sur la surface de la peau, non plus une piqûre, mais une incision dans laquelle on dépose la substance allergène.

Bref, comme on peut le voir, cette notion même de «prick test» ne se caractérise pas par sa clarté et, malgré l'affirmation optimiste du titre de l'ouvrage, l'énigme n'en est pas à sa fin. ce fatras :

C'est finalement l'ouvrage de Fattorusso et Ritter qui jettera quelque lueur sur tout

A. TESTS CUTANÉS DANS L'IMMUNITÉ HUMORALE : ... On utilise en général la technique de la scarification («scratch») ou de la piqûre épicutanée («prick»); l'injection itradermique de l'allergène est réservée à certains allergènes qui n'ont pas donné de résultats clairs avec la technique de la scarification ou de la piqûre...?

Si l'on compare, à l'aide d'un tableau synoptique, les descripteurs du «prick test», tels qu'ils se dégagent des différents énoncés cités plus haut, on se retrouve face à des concordances, il est vrai, mais aussi devant ces divergences qui brouillent considérablement le champ sémantique :

\begin{tabular}{|c|c|c|c|}
\hline Références & $\begin{array}{c}\text { Lieu de dépôt } \\
\text { de la substance }\end{array}$ & $\begin{array}{c}\text { Méthode } \\
\text { utilisée }\end{array}$ & $\begin{array}{c}\text { Appellation du } \\
\text { test }\end{array}$ \\
\hline 1 & - & - & intradermoréaction \\
2 & l'épiderme & la piqûre & - \\
3 & l'épiderme & la piqûre & «prick test» \\
5 & le derme & la piqûre & «prick test» \\
6 & l'épiderme & la scarification & «prick» \\
7 & l'épiderme & la piqûre & \\
\hline
\end{tabular}


Malgré ce manque d'uniformité, on peut, en se basant sur la fréquence relative des concordances et sur le raisonnement, dégager deux faits importants :

1. le «prick test» s'effectue au moyen d'une piqûre (par opposition à la scarification - scratch test).

2. dans le «prick test», la substance allergène est introduite dans l'épiderme (par opposition à l'intradermoréaction, où la substance est introduite dans le derme).

Cependant, la difficulté n'est pas que notionnelle, et ce défrichement partiel du champ sémantique ne permet de clarifier que le signifié. Reste le signifiant, avec tout le chassé-croisé d'équivalents que nous avons vu plus haut.

À première vue, pourtant, la désignation du «prick test» semble aller de soi. Puisque nous savons maintenant que ce test intéresse l'épiderme, que ne l'appelle-t-on «épidermoréaction», sur le modèle de l'intradermoréaction qui désigne le test pratiqué dans le derme? La réponse est simple: la place est déjà prise, si l'on en croit le Dictionnaire français de médecine et de biologie, qui donne épidermoréaction comme synonyme de test épicutané. Or, voici ce qu'il y est dit du test épicutané :

Test destiné à rechercher une hypersensibilité eczémateuse à l'égard d'une substance : celleci est appliquée sur (c'est nous qui mettons en italique) la peau au moyen d'un petit carré de toile, puis protégée par un autre carré de toile imperméable... Syn. : épidermoréaction ${ }^{8}$.

L'expression est donc réservée à un test qui n'est pas le «prick test» et dont l'équivalent anglais est d'ailleurs patch test.

Un autre dictionnaire vient appuyer cette équivalence: «L'épidermo-réaction ( «patch test $\gg$ ) joue un rôle important dans le dépistage étiologique des allergènes provoquant les dermites de contact ${ }^{9} \%$.

On pourrait être tenté, pour trouver une solution au problème, d'appeler le «prick test» cuti-réaction. Deux raisons s'y opposent :

1. ce terme désigne déjà une autre technique voisine mais non identique : la scarification et

2. l'expression cuti-réaction est, comme nous le verrons plus loin, imprécise.

En réalité, pour mieux cerner le problème, il faut élargir l'examen à l'«ensemble» (au sens mathématique) constitué par les tests cutanés. On est alors forcé de faire un constat: il existe plusieurs anomalies dans ce sous-domaine de la terminologie française de l'allergie:

1) Il y a une inadéquation entre les termes anglais et français : comme on l'aura remarqué, l'anglais nomme le test d'après la méthode utilisée, le français d'après la réaction que ce test provoque; ou, plus exactement, d'après la couche de la peau où la réaction a lieu:

—Patch test: test épicutané mais avec, comme synonyme, épidermoréaction ${ }^{8}$

- Scratch test : cuti-réaction 11

- Prick test : intradermoréaction ${ }^{1}$

- Intradermal ou intracutaneous test : intradermoréaction 10 .

Découlent de cette anomalie :

a) une confusion sémantique, comme celle qui apparaît dans certains énoncés français, entre le test et son résultat, et qui donne des effets plutôt inattendus. On peut par exemple lire, dans le Grand dictionnaire encyclopédique Larousse:

La cuti-réaction a vu son champ d'action s'étendre à la recherche de l'allergie en général... ${ }^{12}$ 
Une réaction dont le champ d'action s'étend à la recherche est, pour le moins, bizarre.

Ou encore :

«Le Prick test diffère de la cuti-réaction et de l'intradermoréaction... 13

où l'on compare une technique et deux réactions;

b) une absurdité, dans le cas d'une absence de réaction, c'est-à-dire dans le cas du résultat négatif d'un test. Qu'arrive-t-il en effet si le test ne provoque pas de réaction? On pourrait imaginer, sans vouloir être facétieux, la phrase suivante: la cuti-réaction n'a provoqué aucune réaction !

2) Il existe, au moins dans un cas, une confusion entre le générique et le spécifique. L'expression cuti-réaction, utilisée comme équivalent de scratch test étonne. En réalité, la cuti-réaction (cuti : peau) devrait, comme son étymologie l'indique (et si l'on se résigne à l'assimilation de la réaction au test qui la provoque), désigner toute réaction de la peau, quelle que soit la couche dans laquelle le test est effectué. Autrement dit, que l'on scarifie la peau, qu'on la pique superficiellement ou en profondeur ou encore qu'on applique des bandes de toile à sa surface, la réaction que l'on provoque est en réalité une cuti-réaction. Or, comme on l'a vu, le terme est réservé, sans justification, à la seule scarification.

Pour nous retrouver dans ce labyrinthe, nous avons procédé, en partant de la terminologie anglaise parce qu'elle n'est pas brouillée par la confusion entre test et réaction, à la comparaison du «prick test» avec les autres tests qui lui sont apparentés; et cela, afin de tenter de dégager le ou les traits distinctifs qui permettraient de nommer la notion française de manière monosémique. Nous avons obtenu ce qui suit:

- le patch test consiste à appliquer la substance allergène sur la peau, sans rompre sa continuité;

- le scratch test consiste à scarifier l'épiderme et à y déposer la substance allergène ;

— le prick test consiste à piquer l'épiderme à travers la substance allergène qu'on y aura déposée;

- l'intradermal ou intracutaneous test consiste à piquer dans le derme la substance allergène.

Nous avons ensuite tenté de nommer ces tests, en français, en nous conformant au modèle anglais, c'est-à-dire d'après la méthode utilisée ou d'après la couche de la peau concernée par le test ou, le cas échéant, d'après ces deux descripteurs à la fois. Nous avons obtenu ainsi :

Patch test $=$ test épicutané (épi : sur; cutis : peau).

Scratch test $=$ test de la scarification épidermique, expression que l'on peut, après l'avoir utilisée une première fois in extenso, ramener à test de la scarification.

Prick test $=\quad$ test de la piqûre épidermique, expression que l'on peut, là aussi, après l'avoir utilisée une première fois, ramener à test de la piqûre.

Intradermal ou intracutaneous test $=$ test intradermique. 
Ainsi, après cette triple opération, qui a consisté :

1) à analyser les divers énoncés concernant le «prick test», à mettre en parallèle les descripteurs qu'ils contiennent et à dégager ceux d'entre eux qui sont concordants et qui semblent les plus significatifs;

2) à déterminer le ou les facteurs de confusion de la terminologie française des tests cutanés, auxquels pourrait être attribuable, dans le cas de «prick test», l'équivalence erronée ou le vide terminologique;

3) à tenter de nommer, d'après des descripteurs de même nature, tous les éléments de l'ensemble «tests cutanés»;

nous nous permettons de proposer, pour le terme anglais prick test, la définition et l'équivalent français suivants :

Technique de dépistage de l'allergie, dans laquelle, à travers une goutte de l'allergène déposée sur la peau, on pique l'épiderme à l'aide d'une microlancette que l'on retire ensuite en soulevant légèrement la peau sans la faire saigner. Ce test est utilisé surtout pour le diagnostic de l'hypersensibilité immédiate. La réponse apparaît en général de 10 à 15 minutes après la piqûre et prend la forme de papules érythémateuses.

Équivalent français: Test de la piqûre épidermique

$$
\text { Syn.: Test de la piqûre }
$$

En fait, la démarche que nous avons suivie ici copie modestement celle du clinicien qui, devant une entité pathologique difficile à identifier, procède à ce qu'on appelle un diagnostic différentiel, c'est-à-dire à l'analyse successive de toutes les maladies qui pourraient correspondre à cette entité, et au dégagement des caractéristiques distinctives qui lui sont particulières. Cette démarche pourrait convenir aux recherches terminologiques ponctuelles dans lesquelles les difficultés naissent d'un enchevêtrement des champs sémantiques de notions voisines, ou d'une imprécision dans la désignation de ces notions. Il s'agit en somme d'une démarche comparative qui porte sur les éléments de ce que les mathématiciens appellent «un ensemble ordonné».

Que l'on nous permette, pour conclure, de citer cette phrase par laquelle Jean Hamburger ouvre la préface de la deuxième édition du Dictionnaire de médecine Flammarion:

La langue, c'est la pensée. Mais quand la pensée évolue très vite, la langue suit avec peine. Or, la pensée médicale a subi, depuis plusieurs années, la transformation la plus profonde, le développement le plus étonnant. La langue médicale n'a pas suivi. II a fallu faire du neuf avec des mots qui n'étaient plus de saison, des mots malformés à partir de concepts hasardeux et prématurés. La médecine d'aujourd'hui n'a pas la langue qu'elle mérite.

Le constat est pessimiste mais, assez paradoxalement, roboratif, car il constitue une incitation au travail. Que les terminologues intéressés à la santé de la langue médicale retroussent leurs manches!

AMAL JAMMAL 


\section{NOTES et RÉFÉRENCES}

1. GLADSTONE, W.-J. (1984) : Dictionnaire anglais-français des sciences médicales et paramédicales, $2 \mathrm{e}$ éd., St-Hyacinthe, Québec, Edisem et Paris, Maloine S.A., p. 743.

2. LESSOF, M.H., T.H. LEE, D.M. KEMENY (1987): Allergy: An International Textbook, Baltimore, Williams F. Wilkins, p. 281.

3. JACKSON, W.F. et R. CERIO (1987) : Color Atlas of Allergy, Schering Corporation, U.S.A., p. 17.

4. Ibid.

5. BLACQUE-BÉLAIR, Mathieu DE FOSSEY, M. FOURESTIER : Dictionnaire des constantes biologiques et physiques, $5^{\mathrm{c}}$ éd., Maloine, Paris, p. 826.

6. MICHEL, François-Bernard et Jean BOUSQUET (1986): les Allergies, la fin d'une énigme, Paris, les Guides Hachette, p. 177.

7. FATTORUSSO, V. et O. RITTER (1988): Vademecum clinique, du diagnostic au traitement, $12^{\mathrm{e}}$ éd., Paris, Masson, p. 1204.

8. MANUILA, A., L. MANUILA, M. Nicole et H. LAMBERT (1972) : Dictionnaire français de médecine et de biologie, t. 3, Paris, Masson, p. 930.

9. Dictionnaire des constantes biologiques et physiques, op. cit., p. 831 .

10. Dictionnaire de médecine Flammarion (1989) : $3^{\mathrm{e}}$ éd., Paris, Flammarion Mëdecine-Sciences, p. 448.

11. Dictionnaire des constantes biologiques et physiques, op. cit., p. 824.

12. Grand dictionnaire encyclopédique Larousse (1982) : Paris, p. 2851.

13. JACKSON, W.F. et R. CERIO (1987) : Allergologie en couleurs, traduit en France, publication de Schering Corporation, p. 17. 